Скопје, Македонија

\title{
EQUATIONS OF MOTION OF CLASSICAL NON-RELATIVISTIC STRINGS AND RELATIVISTIC STRINGS
}

\author{
NDRIÇIM SADIKAJ AND ANILA DUKA
}

\begin{abstract}
To formulate the dynamics of a system we can write either the equations of motion or, alternatively, an action. We construct an analytic model describing the macroscopic properties of non-relativistic string and relativistic string. We study the equations of motion for nonrelativistic strings and develop the Lagrangian approach to their dynamics. More importantly, the equations of motion for the relativistic string, the action is a natural generalization of the relativistic particle action. We use the proper area of this surface as the action; this is the Nambu-Goto action. We study the reparameterization property of this action, identify the string tension, and find the equations of motion.
\end{abstract}

\section{INTRODUCTION}

In classical physics, the evolution of a theory is described by its field equations. Suppose we have a non-relativistic point particle, then the field equations for $\mathrm{X}(\mathrm{t})$, i.e. Newtons law $m \ddot{X}(t)=-\frac{\partial V(X(t))}{\partial X(t)}$, follow from extremizing the action, which is given by

$$
S=\int L d t
$$

where $L=T-V$.

\section{EquATIONS OF MOTION FOR TRANSVERSE OSCILLATIONS}

The direction along the string is called the longitudinal direction, and the directions orthogonal to the string are called the transverse directions. Working in the $(x, y)$ plane, let the classical non relativistic string have its endpoints fixed at $(0,0)$, and $(a, 0)$. In a transverse oscillation, the $\mathrm{x}$-coordinate of any point on the string does not change in time. The transverse displacement of a point is given by its y-coordinate.

2000 Mathematics Subject Classification. 05C10, 45654.

Key words and phrases. string theory, oscillation, action, Nambu-Goto action, Lagrangian. 
To describe the classical mechanics of a homogeneous string, we need two pieces of information: the tension $T_{0}$ and the mass per unit length $\mu_{0}$. The total mass of the string is then $M=\mu_{0} \cdot a$.

Consider a small portion of the static string that extends from $\mathrm{x}$ to $\mathrm{x}+$ $\mathrm{dx}$, with $\mathrm{y}=0$. This piece is shown in transverse oscillation in figure.

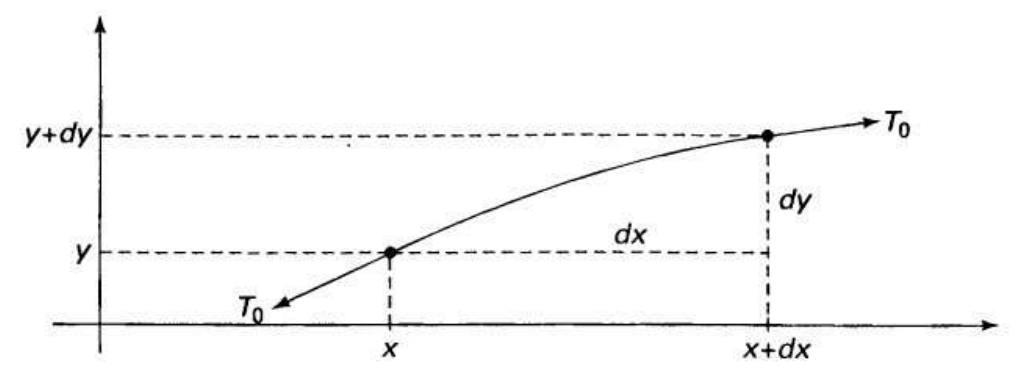

The net vertical force $F_{v}$ is:

$$
d F_{v}=\left.T_{0} \frac{\partial y}{\partial x}\right|_{x+d x}-\left.T_{0} \frac{\partial y}{\partial x}\right|_{x} \cong T_{0} \frac{\partial^{2} y}{\partial x^{2}} d x
$$

By Newton's law, the net vertical force equals mass times vertical acceleration. So,

$$
T_{0} \frac{\partial^{2} y}{\partial x^{2}} d x=\mu_{0} d x \frac{\partial^{2} y}{\partial x^{2}}
$$

We cancel $\mathrm{dx}$ on each side and rearrange terms to get

$$
\frac{\partial^{2} y}{\partial x^{2}}-\frac{\mu_{0}}{T_{0}} \frac{\partial^{2} y}{\partial t^{2}}=0
$$

This is just a wave equation.

The general solution of equation is of the form

$$
y(t, x)=h_{+}\left(x-v_{0} t\right)+h_{-}\left(x+v_{0} t\right)(1)
$$

where $h_{+}$and $h_{-}$are arbitrary functions of a single variable. This solution represents a superposition of two waves, $h_{+}$moving to the right and $h_{-}$moving to the left.

\section{BOUNDARY CONDITIONS AND INITIAL CONDITIONS}

Since equation is a partial differential equation involving space and time derivatives, in order to fix solutions we must in general apply both boundary conditions and initial conditions. Boundary conditions constrain the solution at the boundary of the system, and initial conditions constrain the solution at a given starting time. The most common types of boundary conditions are Dirichlet and Neumann boundary conditions. 
For our string, Dirichlet boundary conditions specify the positions of the string endpoints.

If we attach a massless loop to each end of the string and the loops are allowed to slide along two frictionless poles, we are imposing Neumann boundary conditions.

\section{LAGRANGIAN MECHANICS}

The Lagrangian L of a system is defined by

$$
L=T-V
$$

where $\mathrm{T}$ is the kinetic energy of the system and $\mathrm{V}$ is the potential energy of the system. For a point particle of mass $\mathrm{m}$ moving along the $\mathrm{x}$ axis under the influence of a time-independent potential $\mathrm{V}(\mathrm{x})$, the nonrelativistic Lagrangian takes the form

$$
L(t)=\frac{1}{2} m(\dot{x}(t))^{2}-V(x(t)), \dot{x}(t)=\frac{d x(t)}{d t}
$$

The action $\mathrm{S}$ is defined as

$$
S=\int_{\wp} L(t) d t
$$

where $\wp$ is a path $\mathrm{x}(\mathrm{t})$ between an initial position $x_{i}$ at an initial time $t_{i}$, and a final position $x_{f}$ at a final time $t_{f}$. The action is a functional.

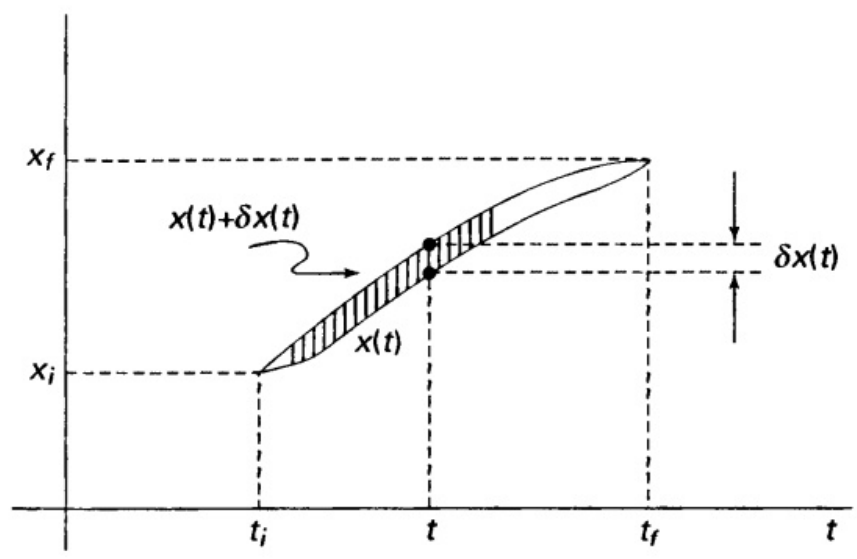

For any path $\mathrm{x}(\mathrm{t})$, the action is given by

$$
S[x]=\int_{t_{i}}^{t_{f}}\left\{\frac{1}{2} m(\dot{x}(t))^{2}-V(x(t))\right\} d t
$$

In terms of the function $\mathrm{x}(\mathrm{t})$ which specifies the path, the perturbed path takes the form $x(t)+\delta x(t)$, as shown in figure 4 . 


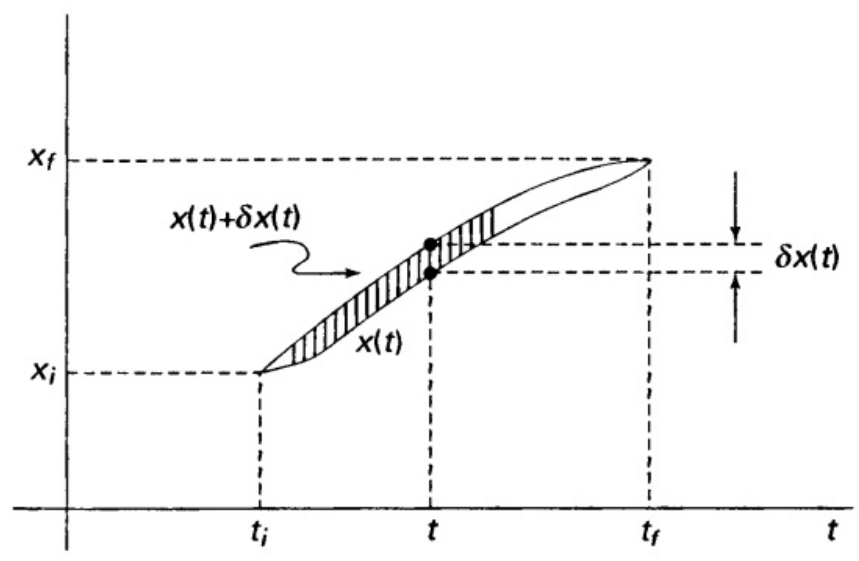

For any time $\mathrm{t}$, the variation $\delta x(t)$ is the vertical distance between the original path and the varied path. As in the figure, we consider variations where the initial and final positions

$$
\delta x\left(t_{i}\right)=\delta x\left(t_{f}\right)=0
$$

The action for the perturbed path :

$$
S[x+\delta x]=\int_{t_{1}}^{t_{f}}\left\{\frac{m}{2}\left(\frac{d}{d t}(x(t)+\delta x(t))\right)^{2}-V(x(t)+\delta x(t))\right\} d t
$$

\section{RELATIVISTIC STRINGS}

We now begin our study of the classical relativistic string-a string that is, in many ways, much more elegant than the non-relativistic one considered before. We focus our attention on the surface traced out by the string in space-time. We use the proper area of this surface as the action; this is the Nambu-Goto action. We study the reparameterization property of this action, identify the string tension, and find the equations of motion.

\section{The Nambu-Goto String action}

Let us now move to our case of interest, the case of surfaces in spacetime. They are two-dimensional and require two parameters.

The proper area is given as

$$
A=\int d \xi^{1} \cdot d \xi^{2} \sqrt{\left(\frac{\partial \vec{x}}{\partial \xi^{1}} \cdot \frac{\partial \vec{x}}{\partial \xi^{1}}\right)\left(\frac{\partial \vec{x}}{\partial \xi^{2}} \cdot \frac{\partial \vec{x}}{\partial \xi^{2}}\right)-\left(\frac{\partial \vec{x}}{\partial \xi^{1}} \cdot \frac{\partial \vec{x}}{\partial \xi^{2}}\right)^{2}}
$$


Now that we are sure that the proper area functional is correctly defined, we can introduce the action for the relativistic string. This action is proportional to the proper area of the world-sheet. To have the units of action we must multiply the area functional by some suitable constants.

The area functional has units of length-squared, as it must be. This is because $X^{\mu}$ has units of length, and each term under the square root has four X. The units of $\tau$ and $\sigma$ cancel out. Each term in the square root has two $\tau$ derivatives and two $\sigma$ derivatives. Their units cancel against the units of the differentials. Nevertheless, we will take $\sigma$ to have units of length and $\tau$ to have units of time.

We do this anticipating a relation between $\tau$ and time and between $\sigma$ and positions on strings. To summarize:

$$
[\tau=T],[\sigma=L],\left[X^{\mu}=L\right],\left[A=L^{2}\right]
$$

Since $\mathrm{S}$ must have units of $M L^{2} / T$ and A has units of $L^{2}$, we must multiply the proper area by a quantity with units of $M / T$. The string tension $T_{0}$ has units of force, and force divided by velocity has the desired units of $M / T$. We can therefore multiply the proper area by $T_{0} / c$ to get a quantity with the units of action. Making use of we set the string action equal to

$$
S=-\frac{T_{0}}{c} \int_{\tau 1}^{\tau f} d \tau \int_{0}^{\sigma 1} d \sigma \sqrt{\left(\dot{X} X^{\prime}\right)^{2}-(\dot{X})^{2}\left(X^{\prime}\right)^{2}}
$$

Here $\sigma>0$ is some constant, and we have introduced some notation for derivatives:

$\dot{X}^{\mu} \equiv \frac{\partial X^{\mu}}{\partial \tau}$ dhe $\left(X^{\mu}\right)^{\prime} \equiv \frac{\partial X^{\mu}}{\partial \sigma}$

The action $\mathrm{S}$ is the Nambu-Goto action for the relativistic string. It is crucial that this action be reparameterization invariant. We can proceed just as we did with spatial surfaces to write the Nambu-Goto action in a manifestly reparameterization invariant way. In this case we have

$$
-d s^{2}=d X^{\mu} d X_{\mid} m u=\eta_{\mu \nu} d X^{\mu} d X^{\nu}=\eta_{\mu \nu} \frac{\partial X^{\mu}}{\partial \xi^{\alpha}} \frac{\partial X^{\nu}}{\partial \xi^{\beta}} d \xi^{\alpha} d \xi^{\beta}
$$

Here $\eta_{\mu \nu}$ is the target-space Minkowski metric. The indices $\alpha$ and $\beta$ run over two values, 1 and 2 , and we have taken $\xi^{1}=\tau$ dhe $\xi^{2}=\sigma$. Just as we did for spatial surfaces, we define an induced metric $\gamma_{\alpha \beta}$ on the world-sheet:

$$
\gamma_{\alpha \beta} \equiv \eta_{\mu \nu} \frac{\partial X^{\mu}}{\partial \xi^{\alpha}} \frac{\partial X^{\nu}}{\partial \xi^{\beta}}=\frac{\partial X}{\partial \xi^{\alpha}} \frac{\partial X}{\partial \xi^{\beta}}
$$

More explicitly, the 2-by-2 matrix $\gamma_{\alpha \beta}$ is

$$
\gamma_{\alpha \beta}=\left[\begin{array}{cc}
(\dot{X})^{2} & \dot{X} \cdot X^{\prime} \\
\dot{X} \cdot X^{\prime} & \left(X^{\prime}\right)^{2}
\end{array}\right]
$$


With the help of this metric we can write the Nambu Goto action in the manifestly reparameterization invariant form

$$
S=-\frac{T_{0}}{c} \int d \tau d \sigma \sqrt{-\gamma}, \gamma=\operatorname{det}\left(\gamma_{\alpha \beta}\right)
$$

In this form, one can readily generalize the NambuGoto action to describe the dynamics of objects that have more dimensions than strings. An action of this kind is useful as a first approximation to the dynamics of D-branes.

\section{Equations of motion}

In this section we will obtain the equations of motion that follow by variation of the string action. In doing so we will also have an opportunity to discuss the various boundary conditions that can be imposed on the ends of open strings. Dirichlet boundary conditions will be interpreted to arise owing to the existence of D-branes.

Let us begin by writing the Nambu-Goto action as the double integral of a Lagrangian density L:

$$
S=\int_{t_{1}}^{t_{f}} d \tau L=\int_{t_{1}}^{t_{f}} d \tau \int_{0}^{\sigma_{1}} d \sigma L\left(\dot{X}^{\mu}, X^{\prime \mu}\right)
$$

where $\mathrm{L}$ is given by

$$
L\left(\dot{X}^{\mu}, X^{\prime \mu}\right)=-\frac{T_{0}}{c} \sqrt{\left(\dot{X} X^{\prime}\right)^{2}-(\dot{X})^{2}\left(X^{\prime}\right)^{2}}
$$

We can obtain the equations of motion for the relativistic string by setting the variation of the action equal to zero. The variation is simply

$$
\delta S=\int_{t_{1}}^{t_{f}} d \tau \int_{0}^{\sigma_{1}} d \sigma\left[\frac{\partial L}{\partial \dot{X}^{\mu}} \frac{\partial\left(\delta X^{\mu}\right)}{\partial \tau}+\frac{\partial L}{\partial X^{\prime \mu}} \frac{\partial\left(\delta X^{\mu}\right)}{\partial \sigma}\right]
$$

where we have used

$$
\delta \dot{X}^{\mu}=\delta\left(\frac{\partial X^{\mu}}{\partial \tau}\right)=\frac{\partial\left(\delta X^{\mu}\right)}{\partial \tau}
$$

and an analogous equation for $\delta X^{\prime \mu}$

The quantities $\frac{\partial L}{\partial \dot{X}^{\mu}}$ and $\frac{\partial L}{\partial X^{\prime \mu}}$ will appear frequently throughout the remainder of our discussion, so it is useful to introduce new symbols for them. This time we find

$$
P_{\mu}^{\tau}=\frac{\partial L}{\partial \dot{X}^{\mu}}=-\frac{T_{0}}{c} \frac{\left(\dot{X} \cdot X^{\prime}\right) X_{\mu}^{\prime}-\left(X^{\prime 2}\right) \dot{X}_{\mu}}{\sqrt{\left(\dot{X} \cdot X^{\prime}\right)^{2}-(\dot{X})^{2}\left(X^{\prime}\right)^{2}}}
$$




$$
P_{\mu}^{\sigma}=\frac{\partial L}{\partial X^{\prime \mu}}=-\frac{T_{0}}{c}=-\frac{T_{0}}{c} \frac{\left(\dot{X} \cdot X^{\prime}\right) \dot{X}_{\mu}-\left(\dot{X}^{2}\right) X_{\mu}^{\prime}}{\sqrt{\left(\dot{X} \cdot X^{\prime}\right)^{2}-(\dot{X})^{2}\left(X^{\prime}\right)^{2}}}
$$

Using this notation $\sigma S$, the variation becomes

$$
\delta S=\int_{t_{1}}^{t_{f}} d \tau \int_{0}^{\sigma_{1}} d \sigma\left[\frac{\partial}{\partial \tau}\left(\delta X^{\mu} P_{\mu}^{\tau}\right)+\frac{\partial}{\partial \sigma}\left(\delta X^{\mu} P_{\mu}^{\sigma}\right)-\delta X^{\mu}\left(\frac{\partial P_{\mu}^{\tau}}{\partial \tau}+\frac{\partial P_{\mu}^{\sigma}}{\partial \sigma}\right)\right]
$$

Since the flow of $\tau$ implies the flow of time, we can imagine specifying the initial and final states of the string, and we restrict ourselves to variations for which $\delta X^{\mu}\left(\tau_{f}, \sigma\right)=\delta X^{\mu}\left(\tau_{i}, \sigma\right)=0$.

We will always assume such variations, so we can forget about these terms. The variation then becomes

$$
\delta S=\int_{t_{1}}^{t_{f}} d \tau\left[\delta X^{\mu} P_{\mu}^{\sigma}\right]_{0}^{\sigma_{1}}-\int_{t_{1}}^{t_{f}} d \tau \int_{0}^{\sigma_{1}} d \sigma \delta X^{\mu}\left(\frac{\partial P_{\mu}^{\tau}}{\partial \tau}+\frac{\partial P_{\mu}^{\sigma}}{\partial \sigma}\right)
$$

Since the second term on the right-hand side must vanish for all variations $\delta X^{\mu}$ of the motion, we set

$$
\frac{\partial P_{\mu}^{\tau}}{\partial \tau}+\frac{\partial P_{\mu}^{\sigma}}{\partial \sigma}=0
$$

This is the equation of motion for the relativistic string, open or closed.

There are two natural boundary conditions that one can impose at an endpoint. The first is a Dirichlet boundary condition, in which the endpoint of the string remains fixed throughout the motion:

$$
\frac{\partial X^{\mu}}{\partial \tau}\left(\tau, \sigma_{*}\right)=0, \mu \neq 0
$$

Since time varies as $\tau$ varies, the value $\mu=0$ must be excluded. Dirichlet boundary conditions are only possible for space directions.

The second possible boundary condition is a free endpoint condition:

$$
P_{\mu}^{\sigma}\left(\tau, \sigma_{*}\right)=0
$$

The boundary conditions can be imposed in many possible ways. For each spatial direction, and at each endpoint, we can choose either a Dirichlet or a free endpoint boundary condition. Since closed strings have no endpoints, they do not require boundary conditions.

Acknowledgements. We thank the Faculty of Technical Sciences of University Ismail Qemali, Vlora, Albania. 


\section{REFERENCES}

[1] Barton Zwiebach, A First Course in String Theory, Second Edition,(2009).

[2] B. M. Barbashov, V. V. Nesterenko, Introduction to the Relativistic String Theory,(1990).

[3] David Tong, String Theory, (2009).

[4] Kevin Wray, An Introduction to String Theory, (2011).

[5] Katerin Becker, Melan Becker, John H.Schwarz, String THEORY AND M-THEORY, A modern introduction,(2007).

Department of Mathematics, Faculty of Technical Sciences, University Ismail Qemali - Vlora, Albania.

E-mail address: ndsadikaj@gmail.com

Department of Mathematics, Faculty of Technical Sciences, University Ismail Qemali - Vlora, Albania.

E-mail address: dukaanila@gmail.com 